\title{
Evaluation of serum and ocular levels of membrane attack complex and C-reactive protein in CFH-genotyped human donors
}

\author{
Kathleen R. Chirco ${ }^{1,2} \cdot$ Miles J. Flamme-Wiese ${ }^{1,2} \cdot$ Jill S. Wiley ${ }^{1,2} \cdot$ Lawrence A. Potempa $^{3}$ Edwin M. Stone ${ }^{1,2}$. \\ Budd A. Tucker ${ }^{1,2} \cdot$ Robert F. Mullins ${ }^{1,2}$
}

Received: 15 June 2018 / Accepted: 24 June 2018 / Published online: 16 July 2018

(c) The Royal College of Ophthalmologists 2018

\begin{abstract}
Background: There is a considerable body of evidence demonstrating a link between the membrane attack complex (MAC) and age-related macular degeneration (AMD), and between C-reactive protein (CRP) and AMD. Both the MAC and the monomeric form of CRP (mCRP) accumulate within the choriocapillaris in AMD. However, the precise contribution of these species to AMD pathophysiology has not been fully elucidated.

Methods: We sought to directly assess CRP and MAC levels between human serum and ocular tissues from the same $C F H$ Y402H genotyped donors using ELISA of serum and RPE/choroid proteins.

Results: The Y402H polymorphism was associated with significantly increased MAC in RPE/choroid samples, but not in the serum, in a previously unstudied cohort. While MAC levels in the choroid were independent of circulating levels, choroidal CRP was correlated to serum levels.

Conclusions: These data provide further evidence for local activation of complement within the choriocapillaris in AMD.
\end{abstract}

\section{Introduction}

A single-nucleotide polymorphism (SNP) in the $\mathrm{CFH}$ gene (rs1061170), which encodes the complement factor $\mathrm{H}(\mathrm{FH})$ protein, increases AMD risk by $\sim 7$-fold in those homozygous for the variant [1]. The monomeric form of Creactive protein (mCRP) and the membrane attack complex of complement (MAC) are both deposited in eyes with AMD and with high-risk CFH genotypes [2, 3]. Here we sought to assess whether the CRP and MAC deposition described in human choroid/Bruch's membrane is due to systemic or local factors by directly comparing circulating and ocular levels of CRP and MAC in the same donors.

Robert F. Mullins

Robert-Mullins@uiowa.edu

1 Institute for Vision Research, The University of Iowa, Iowa City, IA, USA

2 Department of Ophthalmology and Visual Sciences, The University of Iowa, Iowa City, IA, USA

3 Roosevelt University College of Pharmacy, Schaumburg, IL, USA

\section{Methods}

Eyes and post-mortem serum samples from the same donors were obtained from the Iowa Lions Eye Bank (Iowa City, IA). Serum and RPE/choroid tissue from 18 donors was employed for each of the ELISA experiments. All donors were genotyped for the Y402H SNP [3]. Equal amounts of total protein (tissue) or equal volumes of serum were evaluated using ELISA, according to the manufacturer's instructions (CRP Kit, RayBiotech, Norcross, GA; C5b-9 MAC Kit, Quidel, San Diego, CA).

\section{Results}

When CRP levels in RPE/choroid were compared with serum levels from the same donors, a trend toward increased CRP levels for genetically high-risk eyes compared to low-risk eyes was observed, which was consistent with our and others' previous immunohistochemical findings for monomeric CRP [3-5] (Fig. 1a), although this difference in total CRP did not reach statistical significance in the current study. Serum CRP levels did not differ significantly based on $\mathrm{CFH}$ genotype (Fig. 1b). When directly comparing tissue to serum CRP levels for each donor, the 

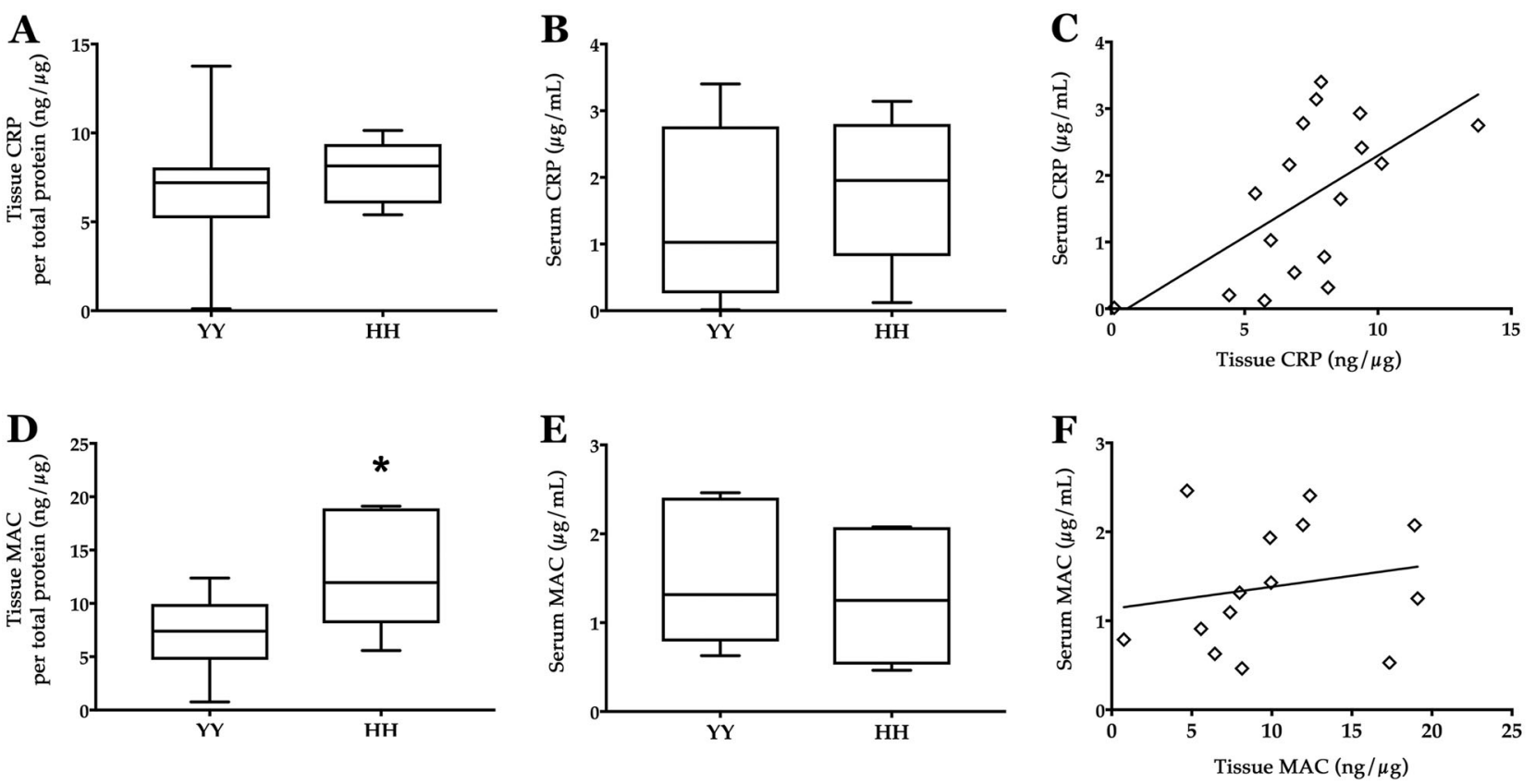

Fig. 1 CRP (a-c) and sC5b-9/MAC (d-f) levels in the RPE/choroid and serum of genotyped human donors. Box-and-whisker plots, showing median, interquartile range, and minimum and maximum values, for RPE/choroid tissue (a, d) and serum (b, e) for low-risk

slope of the regression line was found to be positive and significantly non-zero, suggesting a modest correlation between tissue and serum CRP levels $\left(p<0.05, R^{2}=\right.$ 0.3543; Fig. 1c). These data indicate a weak, but detectable, relationship between CRP levels in tissue and serum from the same individuals. This correlation suggests that the mCRP present in the aging eye is derived from circulating pCRP. This is also consistent with the absence of detectable CRP mRNA in retina, RPE, or choroid (unpublished observations).

MAC levels were found to be significantly elevated $(p<$ $0.05)$ in the RPE/choroid tissue for high-risk compared to low-risk $C F H$ donors (Fig. 1d). Notably, this is the third independent human cohort showing this genotype-phenotype relationship. In contrast, we did not observe a difference in circulating sC5b-9 levels in the serum based on genotype (Fig. 1e). Furthermore, there is no apparent correlation between tissue and serum MAC/sC5b9 levels when directly compared for each donor (Fig. 1f).

\section{Conclusions}

Accumulation of complement complexes in the choroid is a tissue-specific event and is not proportional to circulating complexes. These data suggest that serum levels of the MAC are not related to tissue levels and most likely cannot be used as a predictive measure for AMD or for tissueassociated complement injury in AMD. Taken together,
(YY) and high-risk (HH) $C F H$ donors. Direct comparisons between serum and tissue protein levels reveal a regression line with a significantly non-zero slope for CRP $\left(R^{2}=0.3543\right.$; c) but not for MAC $\left(R^{2}=0.0377 ; \mathbf{f}\right) . * p<0.05$

these data lend further support for the notion that MAC accumulation in AMD is choriocapillaris-specific and that therapies addressing MAC injury to the choroid will be beneficial in AMD.

\section{Summary}

\section{What was known before:}

- CRP and MAC accumulate in aging choriocapillaris.

\section{What this study adds:}

- In the case of CRP, levels are related to circulating CRP in the serum. In the case of MAC, levels in choriocapillaris are independent of those in serum, indicating activation of complement in the local environment.

Acknowledgements We wish to thank the Iowa Lions Eye Bank as well as the donors and their families for their essential contributions to this research. This work was supported in part by NIH grants EY024605 and P30 EY025580, and the Elmer and Sylvia Sramek Charitable Foundation.

Author contributions KRC and RFM designed the experiments, analyzed the data, and wrote the main manuscript text. KRC and JSW performed the experiments. All authors reviewed and edited the manuscript. 


\section{Compliance with ethical standards}

Conflict of interest The authors declare that they have no conflict of interest.

\section{References}

1. Klein RJ, Zeiss C, Chew EY, Tsai JY, Sackler RS, Haynes C, et al. Complement factor $\mathrm{H}$ polymorphism in age-related macular degeneration. Science. 2005;308:385-9.

2. Whitmore SS, Sohn EH, Chirco KR, Drack AV, Stone EM, Tucker $\mathrm{BA}$, et al. Complement activation and choriocapillaris loss in early
AMD: implications for pathophysiology and therapy. Prog Retin Eye Res. 2015;45:1-29.

3. Chirco KR, Whitmore SS, Wang K, Potempa LA, Halder JA, Stone $\mathrm{EM}$, et al. Monomeric C-reactive protein and inflammation in agerelated macular degeneration. J Pathol. 2016;240:173-83.

4. Bhutto IA, Baba T, Merges C, Juriasinghani V, McLeod DS, Lutty GA. C-reactive protein and complement factor $\mathrm{H}$ in aged human eyes and eyes with age-related macular degeneration. Br J Ophthalmol. 2011;95:1323-30.

5. Johnson PT, Betts KE, Radeke MJ, Hageman GS, Anderson DH, Johnson LV. Individuals homozygous for the age-related macular degeneration risk-conferring variant of complement factor $\mathrm{H}$ have elevated levels of CRP in the choroid. Proc Natl Acad Sci USA. 2006;103:17456-61. 